\title{
Transoral robotic-assisted thyroidectomy with central neck dissection: preclinical cadaver feasibility study and proposed surgical technique
}

\author{
Jeremy D. Richmon • F. Christopher Holsinger • \\ Emad Kandil • Michael W. Moore • \\ Jose Armando Garcia $\cdot$ Ralph P. Tufano
}

Received: 23 March 2011 / Accepted: 18 May 2011 / Published online: 15 June 2011

(C) Springer-Verlag London Ltd 2011

\begin{abstract}
Recently, a transoral robotic-assisted technique to access the thyroid gland has been introduced. Despite the advantages this approach may have over other minimally invasive and robotic-assisted techniques, we found that the placement of the camera through the floor of mouth led to restricted freedom of movement. We describe our modification to this technique to overcome this problem. In a study using two fresh human cadavers, the camera port of the da Vinci robot was placed in the midline oral vestibule instead of the floor of the mouth. A transoral thyroidectomy and central neck dissection was successfully performed. Our modification led to an unfettered view of the central neck and allowed for a total thyroidectomy and central neck dissection. Our modification of transoral robotic-assisted thyroidectomy provides superior access to the central compartment of the neck over other robotic-assisted thyroidectomy techniques.
\end{abstract}

F. C. Holsinger contributed equally to the first author.

Electronic supplementary material The online version of this article (doi:10.1007/s11701-011-0287-2) contains supplementary material, which is available to authorized users.

J. D. Richmon · R. P. Tufano $(\bowtie)$

Department of Otolaryngology-Head and Neck Surgery,

Johns Hopkins Hospital, 601 N. Caroline Street 6th Floor,

Blatimore, MD 21287, USA

e-mail: rtufano1@jhmi.edu

E. Kandil

Department of Surgery, Tulane University,

New Orleans, LA, USA

F. C. Holsinger - M. W. Moore - J. A. Garcia

Department of Head and Neck Surgery, The University of Texas

M.D. Anderson Cancer Center, Houston, TX, USA
Keywords Transoral thyroidectomy · Thyroid ·

Robotic-assisted surgery $\cdot$ Robot $\cdot$ Minimally invasive Central neck

\section{Introduction}

Minimally invasive and remote access thyroid surgery continue to evolve and are playing a growing role in the management of benign and select malignant thyroid tumors. Techniques for accessing the thyroid range from minimizing the length and visibility of the cervical scar (minimally-invasive video-assisted thyroidectomy, MIVAT [1-4]) to moving the incision away from the neck altogether to where it is not perceptible (transaxillary endoscopic [5, 6] and robotic-assisted thyroidectomy [7-9]). While these techniques offer various advantages compared to traditional open transcervical thyroidectomy, limitations remain, precluding their widespread adoption for most patients. Currently, approaches must compromise between optimizing cosmesis by hiding scars in the chest and axillary region while maximizing tissue dissection and post-operative pain versus minimizing tissue dissection at the expense of a neck scar. The optimal approach to the thyroid would involve minimal tissue disruption without leaving conspicuous scars. We have recently reported a technique for accessing the central compartment of the neck and removing the thyroid with the da Vinci robot that achieves these goals [10].

Our initial cadaver experience with the da Vinci robot explored a transoral approach with a single port for the camera in the floor of the mouth and two ports for the effector arms in the lateral vestibule under the lip. While this approach was successful, camera mobility was limited by the upper and lower dentition. We present our modifications for 


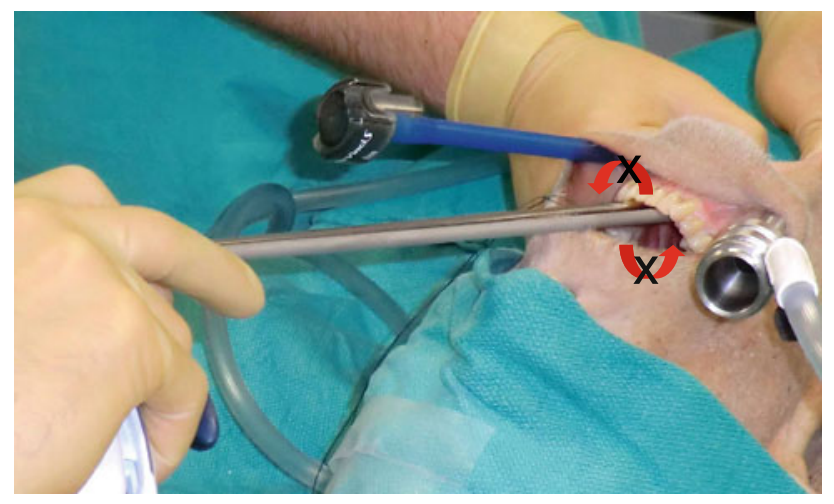

Fig. 1 Original approach to the central neck with the endoscopic camera entering through the floor of the mouth. Rotation along the axis of the scope was precluded by the maxillary and mandibular dentition

overcoming this shortcoming and also look at the feasibility of performing a central neck dissection.

\section{Materials and methods}

Two human cadavers were obtained from the Memorial Hermann Texas Medical Center. One was female and one male, both with full dentition. The procedure was initiated as we had previously described [10] with the neck in slight extension. An incision in the floor of the mouth was made in the midline and a submental subplatysmal pocket was created. Two incisions were made in the gingival-buccal sulcus and each mental nerve was identified and dissected from the surrounding tissue for approximately $2 \mathrm{~cm}$ to avoid tension on the nerve during the procedure. Dissection proceeded around the mandible into the submental pocket. 8 -mm bariatric cannulae were placed in the two lateral incisions and a 12-mm cannula was placed in the floor of mouth incision. Care was taken to place the cannulae posterior to the mental nerves to allow full mobility of the effector arms without applying undue tension on the nerves. The da Vinci robot was brought to the bedside at an angle of approximately $30^{\circ}$ and the cannulae were docked. The $0^{\circ}$ endoscope was advanced through the cannula in the midline floor of the mouth. A Maryland dissector was placed through the left gingival-buccal incision and a Harmonic scalpel was advanced through the right gingival-buccal incision. Under endoscopic visualization, the effector arms were visible; however, the camera motion was limited by its position over the maxillary and under the mandibular dentition. Both the 12 - and $8-\mathrm{mm} 0^{\circ}$ and $30^{\circ}$ scopes were used but adequate freedom of movement to visualize the thyroid bed was not possible due to the dentition (Fig. 1).

Recognizing the limitation of this approach in a population that overwhelmingly has full dentition, we sought an alternative port position for the camera. A third gingival-

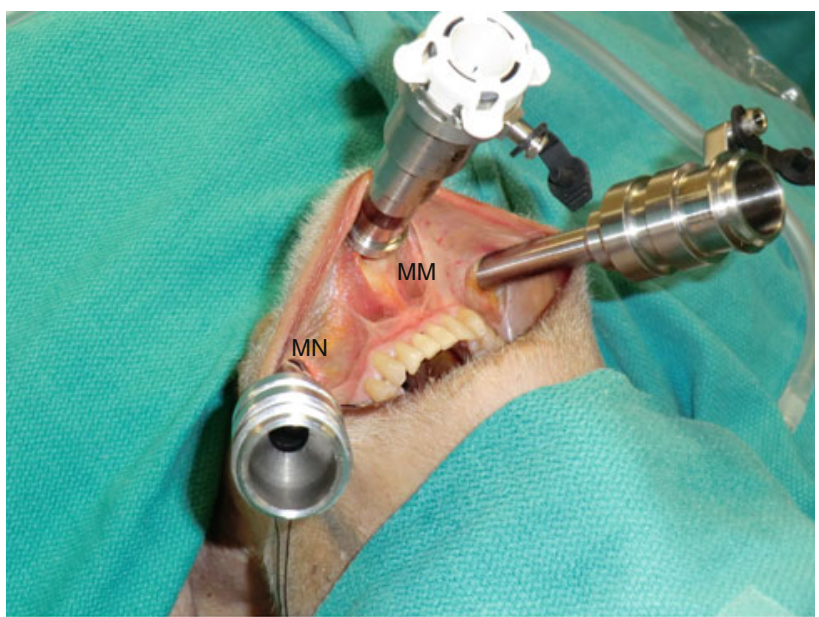

Fig. 2 Placement of the three ports. Each lateral port was positioned posterior to the mental nerve $(M N)$ and the camera port was placed in the midline between the fasiculus of the mentalis muscle $(M M)$

buccal incision was made in the midline, leaving a cuff of mucosa to facilitate closure. The fasciculus of each levator mentalis muscle was identified and the midline was opened. Dissection continued around the inferior aspect of the mandible until the submental pocket was entered. The $12-\mathrm{mm}$ cannula was placed and found to have excellent mobility around the inferior edge of the mandible without any limitation from the dentition (Fig. 2). A $12-\mathrm{mm}^{\circ}$ scope was placed and the robot was docked. $\mathrm{CO}_{2}$ insufflation at $8 \mathrm{~L} / \mathrm{min}$ maintained a working cavity without the need for external retractors (Fig. 3). Superb visualization of the central cavity was obtained. The key steps of the procedure are illustrated in Fig. 4. A brief video is available on-line (LINK). The recurrent laryngeal nerve was identified and preserved, and a right thyroid lobectomy and central neck dissection was performed, the latter as defined by the borders elucidated by the American Thyroid Association's consensus statement on central neck dissection [11]. The inferior border of the dissection was the innominate artery which was easily visualized with this approach. At the conclusion of the procedure, the neck was opened to confirm an appropriate resection (Fig. 5). The approach and dissection were repeated in the second cadaver.

\section{Results and discussion}

We recently described a transoral robotic-assisted thryoidectomy in which the endoscopic port enters the floor of mouth [10]. Although this approach was successful for the previous dentate cadaver, we were unable to maneuver the camera adequately in either of the cadavers used in this dissection due to impingement on the mandibular and maxillary teeth. This limitation, combined with concerns of 

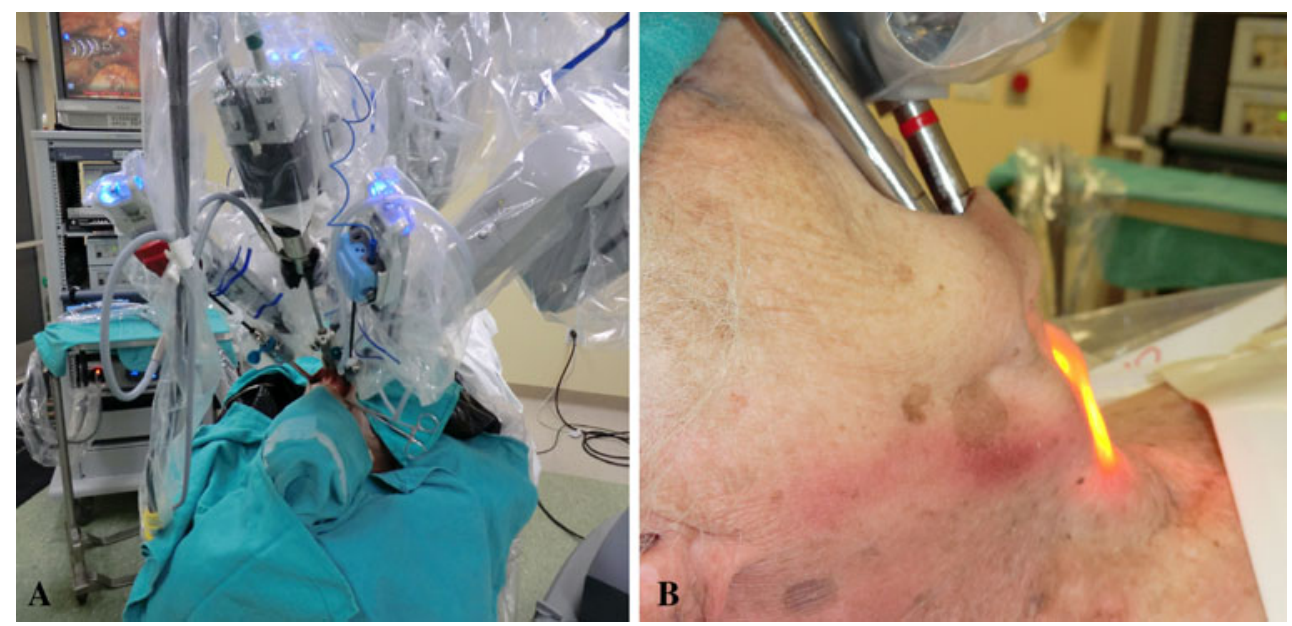

Fig. 3 a Docking of the robot. b A view of the flexed neck demonstrating the insufflated working space and no need for external retractors
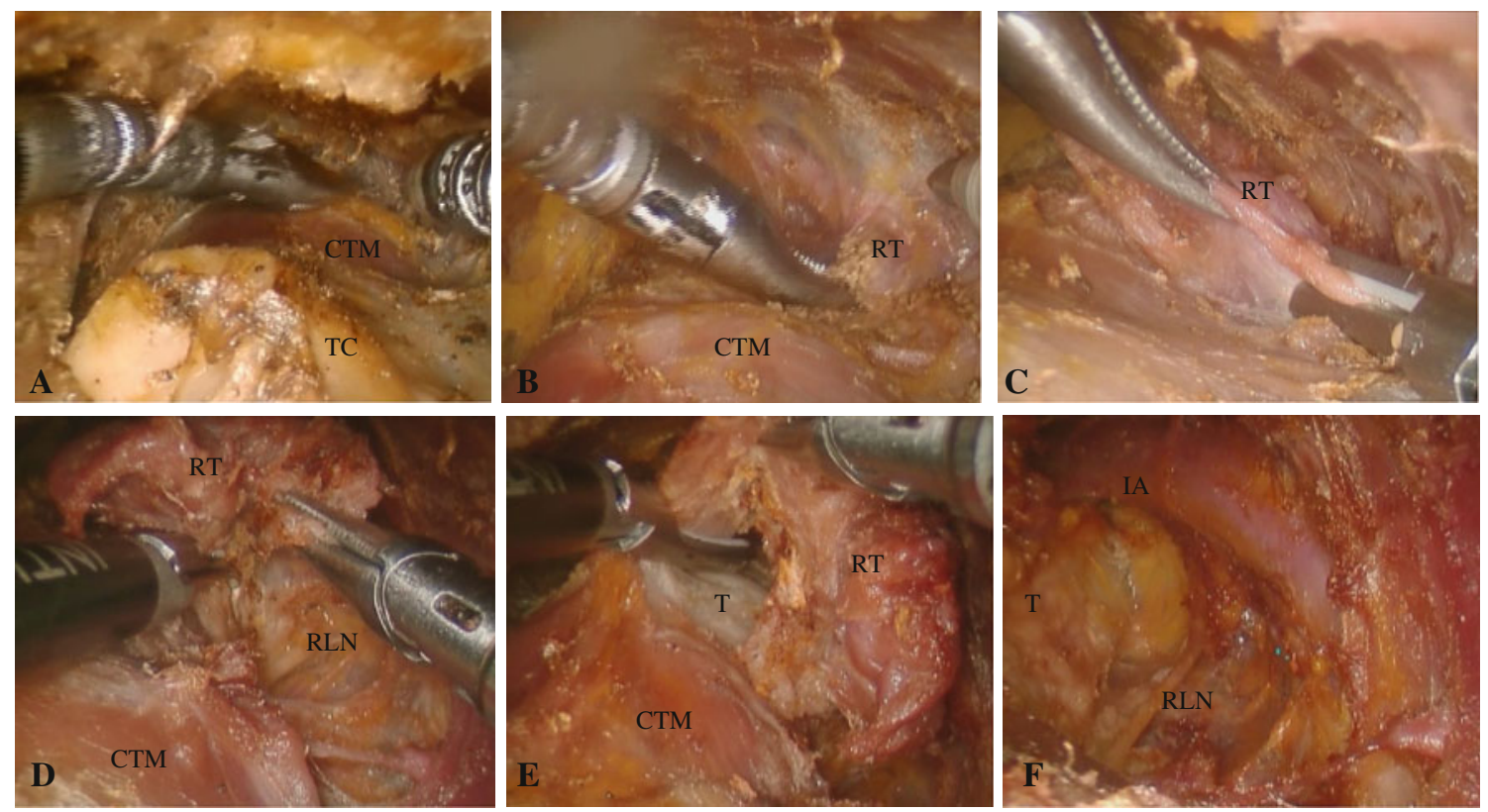

Fig. 4 Various stages of transoral robotic-assisted thyroidectomy with central neck dissection. a Creation of working cavity with $\mathrm{CO}_{2}$ insufflation at $8 \mathrm{~L} / \mathrm{min}$. b Identification of the superior pole of the right thyroid lobe. c Division of the superior thyroid pedical with the Harmonic scalpel. d Elevation of the right thyroid lobe off the trachea and

complications from violating the floor of the mouth, led us to modify the approach. By moving the endoscopic port anterior to the mandible between the mentalis muscles, we were able to obtain excellent mobility without any restriction from the facial skeleton. This modification no longer limits this approach to patients with favorable dentition that would permit passage of the endoscope into the floor of mouth. Furthermore, it should avoid potential deficits in speech and swallowing by avoiding dissection through the tongue and floor of the mouth musculature. However, an anterior midline approach between the mentalis muscles has the potential to result in "puckering" or sagging of the identification of the recurrent laryngeal nerve. e Division of the isthmus. f Central neck after thyroid lobectomy and lymphadenectomy demonstrating innominate artery, recurrent laryngeal nerve and trachea. $T C$ thyroid cartilage, $C T M$ cricothyroid muscle, $R T$ right thyroid lobe, $R L N$ recurrent laryngeal nerve, $T$ trachea, $I A$ innominate artery

mental subunit and cosmetic deformity of the mentum [12, 13]. Furthermore, there remains a risk to the mental nerve with the lateral gingival-buccal port placement. We found that by dissecting the nerve free from the surrounding soft tissue and placing the cannulae posterior to the nerve, there was no tension placed on the nerve during the procedure.

The transoral approach offers superb visibility of the central compartment of the neck down to the level of the innominate artery below the sternal notch. In contrast to other endoscopic and robotic-assisted approaches that have a lateral entry point, the transoral approach provides a midline exposure and equivalent access to both the right and 


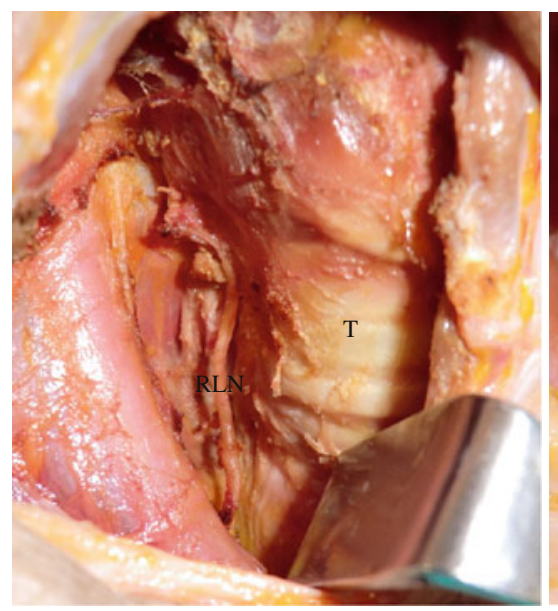

Fig. 5 Post-operative transcervical view of the operative bed demonstrating the area of resection including a right thyroid lobectomy and
Conflict of interest None.

\section{References}

1. Miccoli P et al (2002) Minimally invasive video-assisted thyroidectomy: multiinstitutional experience. World J Surg 26(8):972-975

2. Miccoli P et al (2001) Minimally invasive video-assisted thyroidectomy. Am J Surg 181(6):567-570

3. Miccoli P, Materazzi G (2004) Minimally invasive, video-assisted thyroidectomy (MIVAT). Surg Clin North Am 84(3):735-741

4. Terris DJ, Gourin CG, Chin E (2006) Minimally invasive thyroidectomy: basic and advanced techniques. Laryngoscope $116(3): 350-356$

5. Duncan TD et al (2006) Endoscopic transaxillary near total thyroidectomy. JSLS 10(2):206-211

6. Miyano G, Lobe TE, Wright SK (2008) Bilateral transaxillary endoscopic total thyroidectomy. J Pediatr Surg 43(2):299-303

7. Kang SW et al (2009) Robotic thyroid surgery using a gasless, transaxillary approach and the da Vinci S system: the operative outcomes of 338 consecutive patients. Surgery 146(6):1048-1055

8. Kang SW et al (2009) Robot-assisted endoscopic surgery for thyroid cancer: experience with the first 100 patients. Surg Endosc 23(11):2399-2406

9. Lee J, Kang SW, Jung JJ, Choi UJ, Yun JH, Nam KH, Soh EY, Chung WY (2011) Multicenter study of robotic thyroidectomy: short-term postoperative outcomes and surgeon ergonomic considerations. Ann Surg Oncol. doi:10.1245/s10434-011-1628-0

10. Richmon JD et al (2011) Transoral robotic-assisted thyroidectomy: a preclinical feasibility study in 2 cadavers. Head Neck 33(3):330-333

11. Orloff LA, Kuppersmith RB (2010) American Thyroid Association's central neck dissection terminology and classification for thyroid cancer consensus statement. Otolaryngol Head Neck Surg 142(1):4-5

12. Zide BM, McCarthy J (1989) The mentalis muscle: an essential component of chin and lower lip position. Plast Reconstr Surg 83(3):413-420

13. Chaushu G et al (2001) The effect of precise reattachment of the mentalis muscle on the soft tissue response to genioplasty. J Oral Maxillofac Surg 59(5):510-516

14. Lee J et al (2011) The learning curve for robotic thyroidectomy: a multicenter study. Ann Surg Oncol 18(1):226-232 